\title{
Afterglow calculation in the electromagnetic model for gamma-ray bursts
}

\author{
F. Genet, F. Daigne, and R. Mochkovitch
}

\author{
Institut d'Astrophysique de Paris - UMR 7095 CNRS et Université Pierre et Marie Curie, 98bis boulevard Arago, \\ 75014 Paris, France \\ e-mail: genet@iap.fr
}

Received 30 March 2006 / Accepted 21 May 2006

\section{ABSTRACT}

\begin{abstract}
Aims. We compute the afterglow of gamma-ray bursts produced by purely electromagnetic outflows to see if it shows characteristic signatures differing from those obtained with the standard internal/external shock model.

Methods. Using a simple approach for the injection of electromagnetic energy to the forward shock we obtain the afterglow evolution both during the period of activity of the central source and after. Our method equally applies to a variable source.

Results. Afterglow light curves in the visible and X-ray bands are computed both for a uniform medium and a stellar wind environment. They are brighter at early times than afterglows obtained with the internal/external shock model but relying only on these differences to discriminate between models is not sufficient.
\end{abstract}

Key words. gamma rays: bursts - radiation mechanisms: non-thermal

\section{Introduction}

Lyutikov \& Blandford (2003) proposed an alternative to the standard fireball model where the central engine produces a purely electromagnetic outflow instead of a relativistic baryonic wind. Observationally this electromagnetic model (hereafter EMM) differs from the standard internal/external shock model by the absence of any reverse shock contribution, a different early afterglow evolution and a high polarization of the prompt emission (Lyutikov 2004). In this paper we concentrate on the early afterglow (while the central source is still active) and compare the EMM to the standard model in X-rays and the visible for a uniform external medium or a wind environment. In Sect. 2 we obtain simple equations that govern the evolution of the forward shock propagating in the burst environment. Their solutions are used in Sect. 3 to compute afterglow light curves which are compared to those obtained in the standard model for the same total injected energy. We discuss our results in Sect. 4 and conclude that it likely will be difficult to decide between models from afterglow observations only.

\section{Dynamics of the forward shock}

In the context of the EMM, electromagnetic energy released by the central source directly leads to the formation of a forward shock propagating in the external medium. We obtain the evolution of this forward shock by writing the conservation of energymomentum of the swept-up mass as it accumulates electromagnetic energy

$$
\begin{aligned}
& E_{\mathrm{EM}}+M c^{2}=M \Gamma \Gamma_{\mathrm{i}} c^{2} \\
& E_{\mathrm{EM}}=M \beta \Gamma \Gamma_{\mathrm{i}} c^{2}
\end{aligned}
$$

where $M$ is the swept-up mass, $\beta$ and $\Gamma$ the velocity and Lorentz factor for the bulk motion of the shocked material and $\Gamma_{\mathrm{i}}$ the Lorentz factor for internal motions. The energy $E_{\mathrm{EM}}$ received from the source reads

$E_{\mathrm{EM}}=\int_{0}^{t_{\mathrm{s}}} L_{\mathrm{EM}}(t) \mathrm{d} t$

where $L_{\mathrm{EM}}(t)$ is the source electromagnetic power and $t_{\mathrm{s}}$ the time in the source frame (which is also the observer time modulo the $(1+z)$ factor for time dilation).

This can be easily understood if we define the time $t_{\mathrm{e}}$ when the shocked material is located at radius $R$. The emitted afterglow signal will reach the observer at

$t_{\mathrm{obs}}=t_{\mathrm{e}}+\frac{(D-R)}{c}$

where $D$ is the distance of the source to the observer. At time $t_{\mathrm{e}}$, all power produced by the source up to a time $t_{\mathrm{s}}$ such as

$c\left(t_{\mathrm{e}}-t_{\mathrm{s}}\right)=R$

has been received by the moving shell and Eqs. (3) and (4) then lead to

$t_{\mathrm{obs}}=t_{\mathrm{s}}+\frac{D}{c}$.

Going back to Eq. (1) and after elimination of $\Gamma_{i}$ we get

$2 M c^{2} \Gamma^{2} \sim E_{\mathrm{EM}}$

this approximation being valid in the relativistic phase $(\Gamma \gg 1)$. Differentiation of Eq. (6) with respect to observer time (with the simplified notation $t=t_{\mathrm{obs}}$ ) yields

$L_{\mathrm{EM}}(t)=\frac{16 \pi A c^{2}}{3-s}\left(R^{3-s} \Gamma \frac{\mathrm{d} \Gamma}{\mathrm{d} t}+(3-s) c R^{2-s} \Gamma^{4}\right)$ 

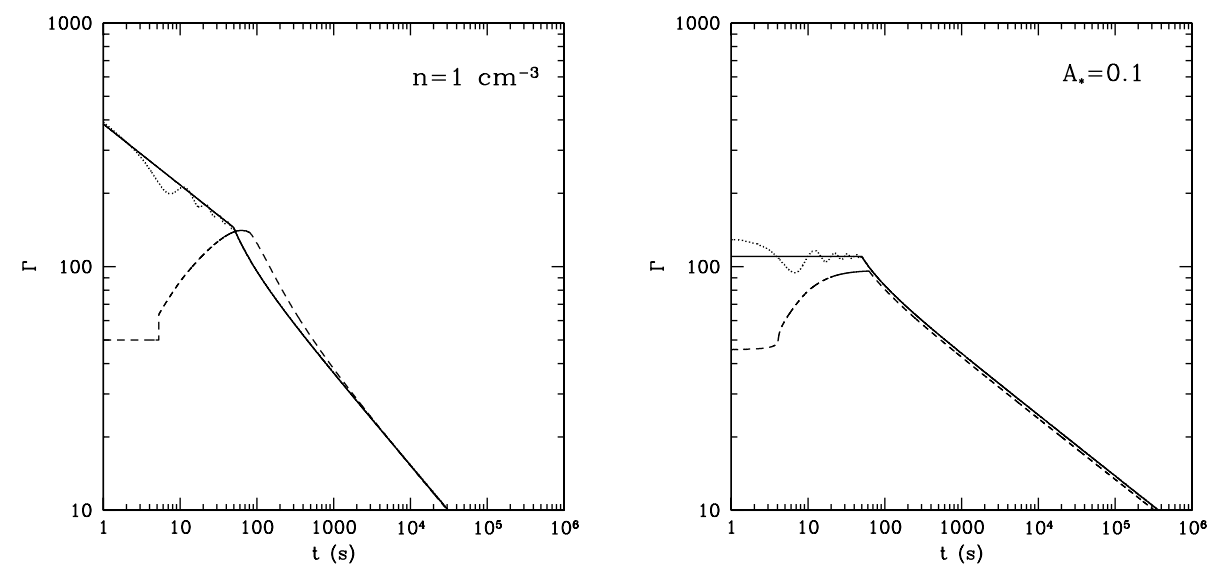

Fig. 1. Evolution of the Lorentz factor in the different models considered; left panel: uniform external medium of density $n=1 \mathrm{~cm}^{-3}$; right panel: stellar wind with $A_{*}=A /\left(5 \times 10^{11} \mathrm{~g} \mathrm{~cm}^{-1}\right)=0.1$. The full, dotted and dashed lines respectively correspond to the EMM with a constant $L_{\mathrm{EM}}=10^{52} \mathrm{erg} \mathrm{s}^{-1}$, a variable $L_{\mathrm{EM}}(t)$ given by Eq. (13) and to the standard internal/external shock model with $\dot{E}=10^{52} \mathrm{erg} \mathrm{s}^{-1}$ and an initial distribution of the Lorentz factor in the relativistic wind given by Eq. (14).

where we have written

$M(R)=\frac{4 \pi A}{3-s} R^{3-s}$

with $A=\rho$ and $s=0$ for a uniform medium of density $\rho$ and $A=5 \times 10^{11} A_{*} \mathrm{~g} \mathrm{~cm}^{-1}$ and $s=2$ for a wind environment. With the additional relation between observer time and shock radius

$\frac{\mathrm{d} R}{\mathrm{~d} t}=2 c \Gamma^{2}$

the problem can be solved for any law $L_{\mathrm{EM}}(t)$. With a constant $L_{\mathrm{EM}}$ it can be easily shown that the solutions of Eqs. (7) and (9) are

$\Gamma=(Q / 2)^{1 / 2}(c t)^{-1 / 4} \quad$ and $\quad R=2 Q(c t)^{1 / 2}$

with

$Q=\left(\frac{3 L_{\mathrm{EM}}}{32 \pi \rho c^{3}}\right)^{1 / 4}$

for a uniform medium and

$\Gamma=\left(\frac{L_{\mathrm{EM}}}{16 \pi A c^{3}}\right)^{1 / 4}$ and $R=2 c \Gamma^{2} t$

for a stellar wind. With a variable $L_{\mathrm{EM}}(t)$, Eqs. (7) and (9) are integrated using Eqs. (10) or (12) as initial conditions with $L_{\mathrm{EM}}=L_{\mathrm{EM}}(t=0)$. Figure 1 shows the resulting $\Gamma(t)$ for a constant $L_{\mathrm{EM}}=10^{52} \mathrm{erg} \mathrm{s}^{-1}$ or $L_{\mathrm{EM}}(t)$ given by

$L_{\mathrm{EM}}(t)=10^{52}\left[1+\cos 2 \pi\left(\frac{t}{10 \mathrm{~s}}\right)\right] \operatorname{erg~s}^{-1}$.

In both cases the source is supposed to be active for $50 \mathrm{~s}$. For comparison we also plot in Fig. 1 the evolution of the Lorentz factor computed in the standard internal/external shock model. In that case, we assume that a constant kinetic power $\dot{E}=10^{52} \mathrm{erg} \mathrm{s}^{-1}$ is injected in the relativistic wind for a duration $t_{\mathrm{W}}=50 \mathrm{~s}$. We consider a single pulse burst produced by a distribution of the Lorentz factor of the form

$\Gamma(t)=\frac{\Gamma_{\max }+\Gamma_{\min }}{2}-\frac{\Gamma_{\max }-\Gamma_{\min }}{2} \cos \left(\pi \frac{t}{0.2 t_{\mathrm{W}}}\right)$

if $t<0.2 t_{\mathrm{W}}$ and $\Gamma(t)=\Gamma_{\max }$ if $t>0.2 t_{\mathrm{W}} ; \Gamma_{\max }=200$ and $\Gamma_{\min }=50$ are the maximum and minimum values of the
Lorentz factor so that the most rapid part of the wind is decelerated by the slower one which was emitted previously. To follow the wind evolution and its interaction with the external medium we represent the flow with a large number $(\gtrsim 1000)$ of discrete shells which interact by direct collision only, pressure waves being neglected (Daigne \& Mochkovitch 1998). The action of the external medium is included by progressively adding the swept-up mass to the front shell of the wind. In all cases, the Lorentz factor relaxes to the standard Blandford-McKee (1976) solution after a few $10^{2} \mathrm{~s}$. However at early times $\Gamma$ is larger in the EMM, especially for a uniform external medium.

\section{Afterglow calculation}

\subsection{Method}

Using the dynamical evolution of the forward shock obtained above we have calculated afterglow lightcurves in X-rays and in the $V$ band for both the electromagnetic and the standard model. We obtain the two critical frequencies $v_{\mathrm{m}}$ and $v_{\mathrm{c}}$ following Sari et al. (1998). They are represented in Fig. 2 for a uniform medium and a stellar wind environment. In the EMM with a uniform medium and a constant source, the ratio $v_{\mathrm{m}} / v_{\mathrm{c}}$ is given by

$\frac{v_{\mathrm{m}}}{v_{\mathrm{c}}}=0.065 \epsilon_{\mathrm{e},-1}^{2} \epsilon_{B,-3}^{2} n L_{52}$

where $n$ is the density in $\mathrm{cm}^{-3}, L_{52}$ the electromagnetic power in units of $10^{52} \mathrm{erg} \mathrm{s}^{-1}, \epsilon_{\mathrm{e},-1}=\epsilon_{\mathrm{e}} / 10^{-1}$ and $\epsilon_{B,-3}=\epsilon_{B} / 10^{-3}$ (the numerical factor in Eq. (15) corresponding to the electron power law index $p=2.5$ ). The cooling regime therefore remains fixed as long as the source is active (and does not vary). In Fig. 2 we have adopted $\epsilon_{\mathrm{e},-1}=\epsilon_{B,-3}=L_{52}=1$ and $n=1$ or $A_{*}=0.1$ in the uniform medium or wind case respectively.

With these parameters the afterglow is always in the slow cooling regime in the uniform medium case while in the wind case it is in fast cooling while the source is active and moves to slow cooling shortly after.

\subsection{Results}

We have represented in Fig. 3 afterglow light curves in X-rays (2-10 keV) and in the $V$ band for a uniform external medium and a stellar wind. The burst parameters are identical to those used 

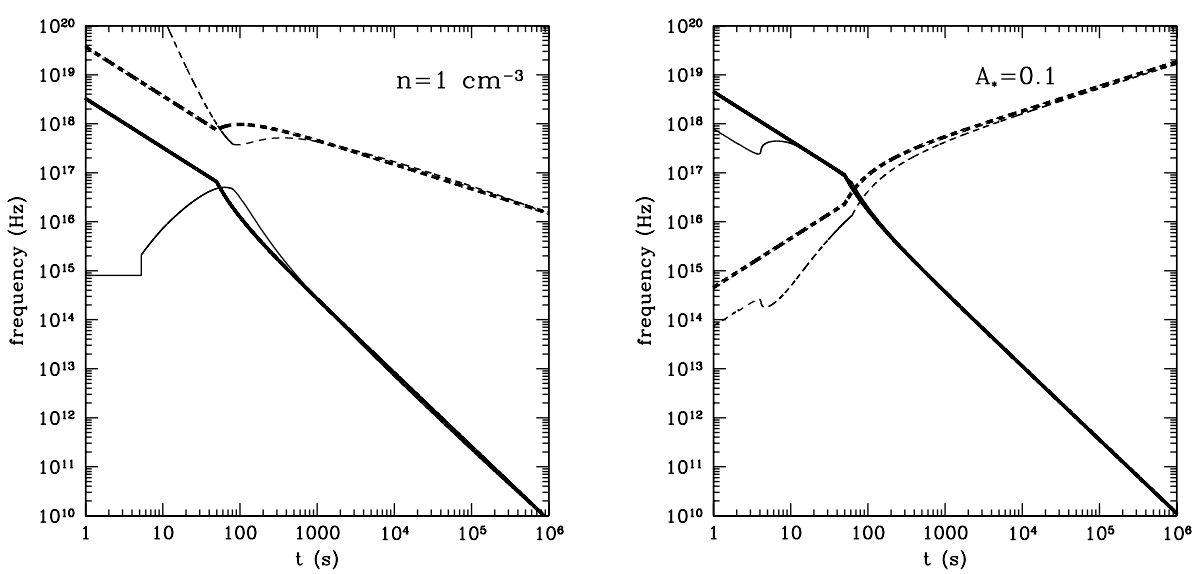

Fig. 2. The two frequencies $v_{\mathrm{m}}$ (full lines) and $v_{\mathrm{c}}$ (dashed lines) in the EMM (thick lines) and the standard model (thin lines); left panel: uniform external medium of density $n=1 \mathrm{~cm}^{-3}$; right panel: stellar wind with $A_{*}=0.1$.
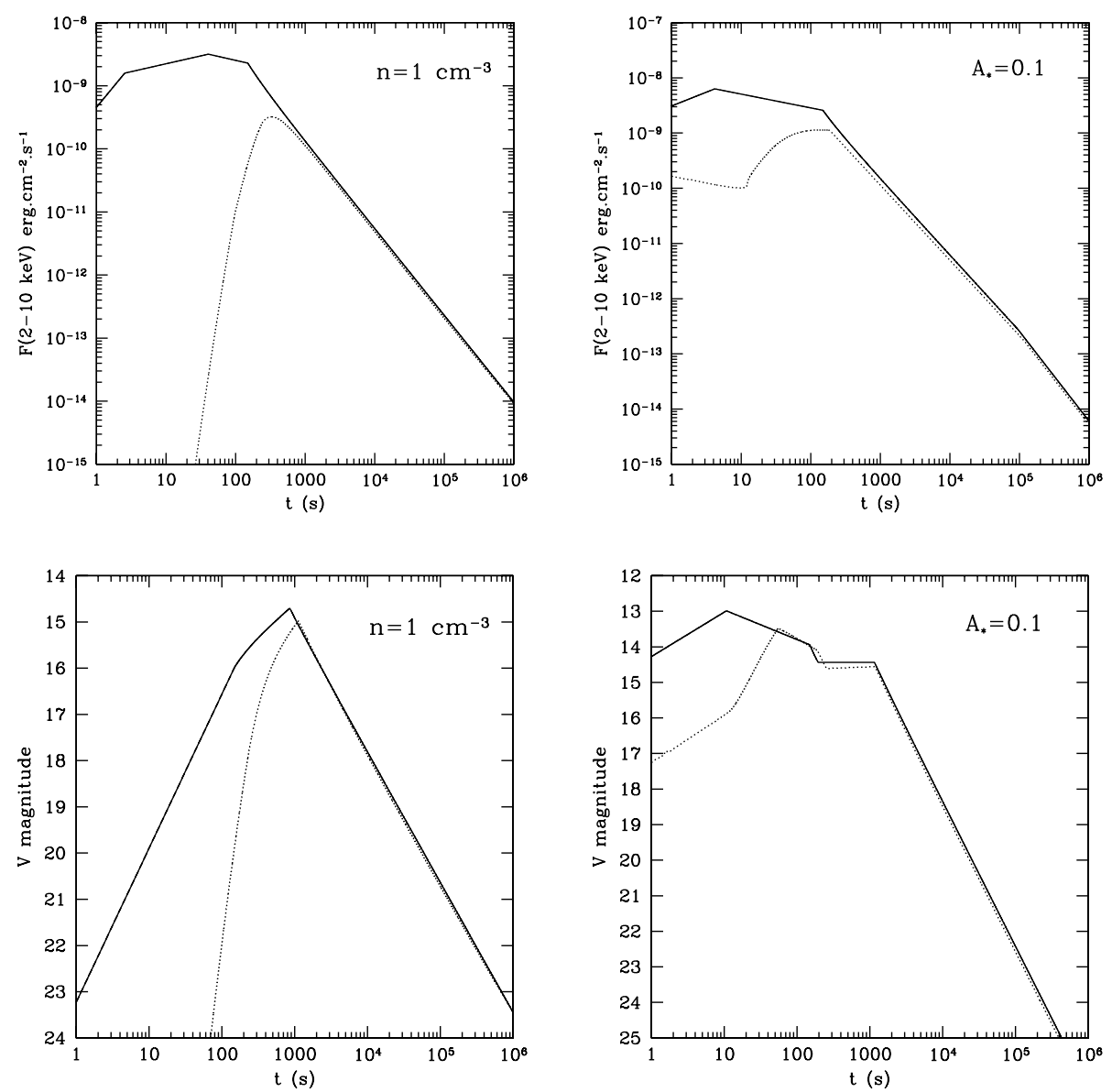

Fig. 3. Afterglow comparison between the EMM (solid line) and the standard model (dotted line). Top: X-ray band (2-10 keV); bottom: $V$ band; left column: uniform medium of density $n=1 \mathrm{~cm}^{-3}$; right column: stellar wind with $A_{*}=0.1$. A redshift $z=2$ has been assumed.

in Fig. 2. For comparison we also show in Fig. 3 standard afterglows computed in the internal/external shock model with the same total injected energy. A redshift $z=2$, typical of SWIFT bursts has been adopted (Jakobsson et al. 2006). In all cases the afterglows are brighter at early times in the EMM while at late times (in the Blandford-McKee regime) the models coincide. The difference in early evolution is larger for a uniform external medium, especially in X-rays. This is due to the large initial Lorentz factor in the EMM compared to the standard model (see Fig. 1) which leads to a higher electron Lorentz factor in the shocked external medium. The frequency $v_{\mathrm{m}}$ is then in the X-ray band already at very early times while it is still in the UV/visible in the standard model. In the wind case, the differences between the EMM and the standard case are smaller and the two models will be therefore more difficult to distinguish.

For the EMM we have also calculated afterglows when the source is variable (with $L_{\mathrm{EM}}(t)$ given by Eq. (13)). The results are shown in Fig. 4 for a uniform external medium and the first $200 \mathrm{~s}$ of evolution (the source being active for $150 \mathrm{~s}$ in the observer frame). The left panel in Fig. 4 shows the simple calculation which only includes line of sight emission. It produces a highly variable X-ray light curve but off-axis effects (time delays and 

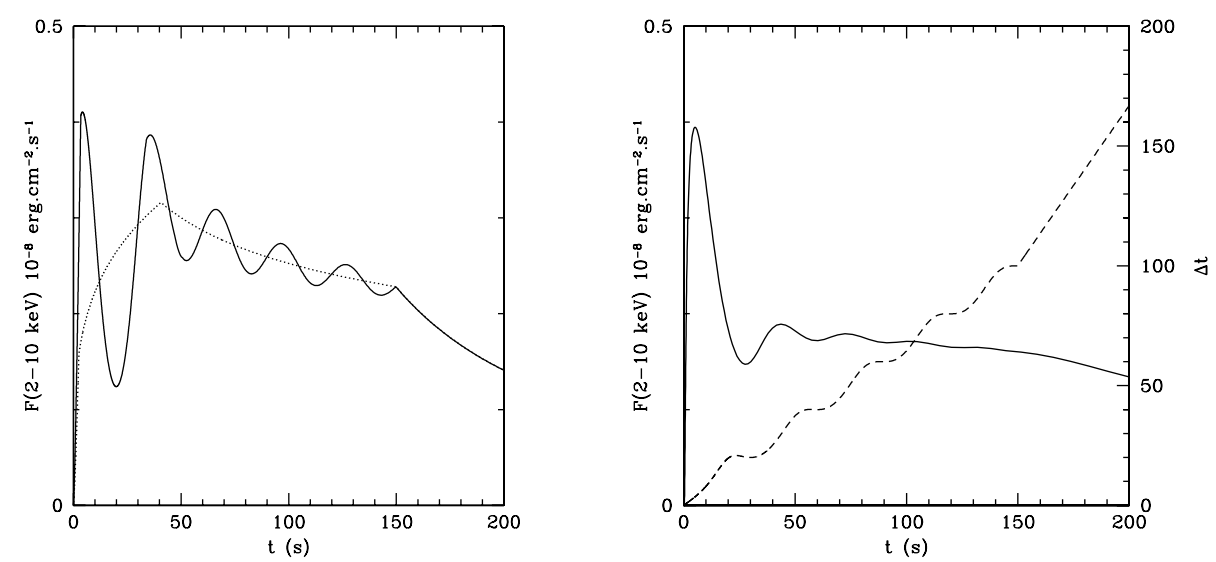

Fig. 4. Early afterglows for a variable source $\left(L_{\mathrm{EM}}(t)\right.$ being given by Eq. (13)). Left panel: afterglow computed with on-axis emission only compared to the case of a constant source (dotted line). Right panel: afterglow computed with off-axis emission included (full line) and geometrical delay $\Delta t=R / 2 c \Gamma^{2}$ (dashed line). A uniform external medium of density $n=1 \mathrm{~cm}^{-3}$ and a redshift $z=2$ have been assumed.

spectral softening) will smear out any variability occurring on a time scale shorter than

$\Delta t=\frac{R}{2 c \Gamma^{2}}$

where $R$ and $\Gamma$ are the radius and Lorentz factor of the emitting shell. The right panel of Fig. 4 shows $\Delta t$ as a function of observer time together with the X-ray light curve now obtained with a detailed calculation including off-axis effects (Granot et al. 1999; Woods \& Loeb 1999). Except for the first two or three peaks which partially subsist because $\Delta t$ is initially small, the rest of the curve becomes nearly monotonic. In the optical the variability is barely visible, even without including off-axis effects.

\section{Discussion and conclusion}

The lightcurves in Fig. 3 show that the EMM and the standard model notably differ at early times (during the period of source activity). However relying on these differences alone to identify the physical origin of GRBs will be a difficult task requiring a very early follow-up of the afterglow. In X-rays, SWIFT should be able to do that (at least in some cases) but the problem here will come from the mixing of the afterglow contribution with the brighter prompt emission component. This mixing will also probably prevent an unambiguous detection of the imprint of source variability on the X-ray afterglow (Fig. 4). In the visible, where the burst prompt emission is weak and probably negligible (see however the recent RAPTOR observations of
GRB 041219a and GRB 050820a (Vestrand et al. 2005, 2006)), the EMM predicts a brighter afterglow for a given set of parameters $\epsilon_{\mathrm{e}}, \epsilon_{B}, n$ or $A_{*}$. But in real afterglows these parameters are not known a priori and deciding between models will be tricky. Polarization properties of the burst prompt emission (Lyutikov 2004) when they become more easily accessible may provide clearer evidence.

A last interesting point concerning the EMM is related to the shallow part observed by SWIFT in many X-ray afterglows. The light curves in Fig. 3 show that the EMM indeed predicts an initially flat region in the early X-ray afterglow. However this flat region does not last more than the period of source activity (150 $\mathrm{s}$ in observer time in Fig. 3). It would then extend to $10^{4} \mathrm{~s}$ (or more) only if the source can remain active for that duration, as was also suggested for the standard model (Zhang et al. 2005).

\section{References}

Blandford, R. D., \& McKee, C. F. 1976, PhFl, 19, 1130

Daigne, F., \& Mochkovitch, R. 1998, MNRAS, 296, 275

Granot, J., Piran, T. \& Sari, R. 1999, ApJ, 513, 679.

Jakobsson, P., Levan, A., Fynbo, J. P. U., et al. 2006, A\&A, 447, 897

Lyutikov, M., \& Blandford, R. D. 2003 [arXiv: astro-ph/0312347]

Lyutikov, M. 2004, 35th COSPAR Meeting [arXiv: astro-ph/0409489]

Sari, R., Piran, T., \& Narayan, R. 1998, ApJ, 497, L17

Vestrand, W. T., Wozniak, P. R., Wren, J. A., et al. 2005, Nature, 435, 178

Vestrand, W. T., Wren, J. A., Wozniak, P. R. et al. 2006, to appear in Nature

Woods, E., \& Loeb, A. 1999, ApJ, 523, 187

Zhang, B., Fan, Y. Z., Dyks, J., et al. 2006, ApJ, 642, 354 